PROCEEDINGS OF THE

AMERICAN MATHEMATICAL SOCIETY

Volume 127, Number 11, Pages 3191-3200

S 0002-9939(99)04908-4

Article electronically published on April 27, 1999

\title{
REGULARITY OF CR MAPS BETWEEN CONVEX HYPERSURFACES OF FINITE TYPE
}

\author{
BERNARD COUPET, HERVÉ GAUSSIER, AND ALEXANDRE SUKHOV \\ (Communicated by Steven R. Bell)
}

\begin{abstract}
We study rigidity and regularity properties of $\mathrm{CR}$ maps between smooth convex hypersurfaces of finite type in $\mathbb{C}^{n+1}$.
\end{abstract}

\section{INTRODUCTION}

The present paper is devoted to the study of regularity of Cauchy-Riemann (CR) maps. More precisely, let $f: \Gamma_{1} \rightarrow \Gamma_{2}$ be a continuous CR map between two smooth pseudoconvex hypersurfaces $\Gamma_{1}$ and $\Gamma_{2}$ of finite type in the sense of D'Angelo. Under what conditions is $f$ smooth? The strictly pseudoconvex case was considered by L.Nirenberg, S.Webster and P.Yang [13] and by S.Pinchuk and Sh.Tsyganov [15]. In the finite type case S.Bell [3] proved the regularity of CR homeomorphisms; this result was extended by S.Bell and D.Catlin [4] to the class of CR maps which possess some finiteness properties. They showed that if for some point $p \in \Gamma_{1}$ the pullback $f^{-1}(f(p))$ is compact in $\Gamma_{1}$, then $f$ is smooth and locally finite near $p$ (in fact the former condition allows us to extend $f$ as a proper holomorphic map between suitable one-sided neighborhoods of $\Gamma_{j}$ and apply the well-developed techniques of the Bergman projection). The main purpose of our paper is to show that in the case where the hypersurfaces are convex this compactness condition automatically holds if the map $f$ is supposed to be just nonconstant with minimal regularity assumptions.

Our main result is the following:

Theorem A. Let $f: \Gamma_{1} \rightarrow \Gamma_{2}$ be a nonconstant continuous CR map between two $\mathcal{C}^{\infty}$ smooth convex hypersurfaces in $\mathbb{C}^{n+1}$ of finite type in the sense of D'Angelo near points $p$ and $f(p)$ respectively. Assume that at least one of the following conditions holds:

(i) $f$ is a Lipschitz map,

(ii) $\Gamma_{1}$ is strictly pseudoconvex at $p$.

Then the pullback $f^{-1}(f(p))$ is compact near $p$ in $\Gamma_{1}$.

In view of [4] we have the following corollaries:

Received by the editors January 15, 1998.

1991 Mathematics Subject Classification. Primary 32H40, 32H99; Secondary 32F40, 32G07, 32H15, 32H35, 32M99.

Key words and phrases. Finite type, scaling method, CR map, boundary regularity.

(C)1999 American Mathematical Society 
Corollary B. Any Lipschitz continuous $C R$ map between two $\mathcal{C}^{\infty}$ smooth convex hypersurfaces of finite type in the sense of D'Angelo in $\mathbb{C}^{n+1}$ is $\mathcal{C}^{\infty}$ smooth.

Corollary C. Let $f: \Gamma_{1} \rightarrow \Gamma_{2}$ be a nonconstant continuous CR map between two $\mathcal{C}^{\infty}$ smooth hypersurfaces in $\mathbb{C}^{n+1}$. Assume that $\Gamma_{1}$ is strictly pseudoconvex near a point $p$ and $\Gamma_{2}$ is convex of finite type in the sense of D'Angelo near $f(p)$. Then $f$ is a $\mathcal{C}^{\infty}$ smooth local diffeomorphism and $\Gamma_{2}$ is strictly pseudoconvex near $f(p)$.

Our basic idea is to use the scaling method which allows us to reduce the consideration of the general case to the investigation of simpler CR structures on algebraic hypersurfaces.

The paper is divided in three sections. In the first section we recall the basic scaling construction, we give uniform estimates on the dilated maps and we end with some basic properties of the limit map; this allows us to prove part (i) of Theorem A in the second section. In the third section we give the proof of part (ii) of Theorem A, based on the study of boundary behavior of the limit map.

If $f_{\nu, \zeta}(z)$ and $g_{\nu, \zeta}(z)$ are (sequences of) functions, we will write $f_{\nu, \zeta}(z) \lesssim g_{\nu, \zeta}(z)$ if there is a positive constant $C$ independent of all variables and parameters such that $f_{\nu, \zeta}(z) \leq C g_{\nu, \zeta}(z)$ for all $z, \nu, \zeta$, and $f_{\nu, \zeta}(z) \simeq g_{\nu, \zeta}(z)$ if $f_{\nu, \zeta}(z) \lesssim g_{\nu, \zeta}(z) \lesssim f_{\nu, \zeta}(z)$ for all $z, \nu, \zeta$.

This paper is a revised version of our preprint On local version of Fefferman's mapping theorem (1996).

\section{Scaling of the Domains $\Omega_{1}$ AND $\Omega_{2}$}

In the hypothesis of Theorem A we have the following distance equivalence property:

Proposition 1.1. For every compact set $K$ in $\Gamma_{1}$ there exists a neighborhood $U$ of $K$ such that for every $z$ in $\Omega_{1} \cap U$ :

$$
d\left(z, \Gamma_{1}\right) \lesssim d\left(f(z), \Gamma_{2}\right) \lesssim d\left(z, \Gamma_{1}\right)
$$

where $d(.,$.$) denote the euclidean distance.$

Proof of Proposition 1.1. Since the defining function $r_{2}$ of $\Gamma_{2}$ is convex and, in particular, plurisubharmonic on $\Omega_{2}$, it suffices to apply the Hopf lemma to the composition $r_{2} \circ f$ in order to get the left inequality. The right one is immediate if $f$ is Lipschitz up to the boundary; we refer the reader to [6] and [17] for the case where $\Gamma_{1}$ is strictly pseudoconvex.

Let $f$ be a nonconstant continuous CR map between a convex hypersurface $\Gamma_{1}$ of finite type $2 k$ and a convex hypersurface $\Gamma_{2}$ of finite type $2 m$ satisfying $f(0)=0$. One can suppose that $f$ is extended holomorphically to the convex side $\Omega_{1}$ of $\Gamma_{1}$, maps it to the convex side $\Omega_{2}$ of $\Gamma_{2}$ ([3]) and is continuous up to $\Gamma_{1}$ with $f\left(\Gamma_{1}\right) \subset \Gamma_{2}$. Let $\left(p^{\nu}\right)_{\nu}$ be a sequence of points in $\Omega_{1}$ on the real normal at $0 \in \Gamma_{1}$ and converging to 0 . Since $\Omega_{1}$ is convex of finite type $2 k$, according to [8] one can choose holomorphic local coordinates such that $\Omega_{1}$ is defined by the function $r_{1}$ of the form:

$$
r_{1}(z)=\operatorname{Im} z_{0}+H\left(z^{\prime}\right)+R_{1}\left(z^{\prime}\right)+\left(\operatorname{Re} z_{0}\right) R_{2}\left(z^{\prime}\right)+R_{3}(z)
$$


where:

(a) $H$ is a real-valued plurisubharmonic polynomial without pluriharmonic terms in the variables $z^{\prime}$ of weighted degree 1 with respect to $\left(m_{1}, \ldots, m_{n}\right)\left(m_{j}\right.$ being positive integers).

(b) $\left|R_{1}\left(z^{\prime}\right)\right| \leq C\left(\sum_{j=1}^{n}\left|z_{j}\right|^{m_{j}}\right)^{1+\delta}$ for suitable positive constants $C, \delta$.

(c) $\left|R_{2}\left(z^{\prime}\right)\right| \leq C\left|z^{\prime}\right|,\left|R_{3}\left(z^{\prime}\right)\right| \leq C\left(\operatorname{Re} z_{0}\right)^{2}$.

(d) 0 is a point of finite type of $\Omega_{0}=\left\{\operatorname{Im} z_{0}+H\left(z^{\prime}\right)<0\right\}$.

We consider the sequence $\left(p^{\nu}=\left(-\delta_{\nu} i, 0^{\prime}\right)\right)_{\nu}$ in $\Omega_{1}$, where $\delta_{\nu}=1 / \nu$ and the sequence $\left(q^{\nu}=f\left(p^{\nu}\right)\right)_{\nu}$ in $\Omega_{2}$.

For the domain $\Omega_{1}$ we define the dilations

$$
\Lambda_{1, \nu}(z)=\left(\left(\delta_{\nu}\right) z_{0},\left(\delta_{\nu}\right)^{m_{1}} z_{1}, \ldots,\left(\delta_{\nu}\right)^{m_{n}} z_{n}\right)
$$

and the dilated domains:

$$
\Omega_{1}^{\nu}=\left\{z \in\left(\Lambda_{1, \nu}\right)^{-1}\left(U_{1}\right): \delta_{\nu}^{-1} r_{1} \circ \Lambda_{1, \nu}(z)<0\right\} .
$$

The scaling of $\Omega_{2}$ is more delicate. The convexity of the dilated family of domains is important for uniform estimates of the Kobayashi metric (see Proposition 1.3); we need the convexity of the limit domain in order to study the properties of the limit map (see Lemma 1.1 below) as well. For these reasons we use the version of the scaling developed in [10] and scale $\Omega_{2}$ by a sequence of affine maps conserving the convexity (compare with [2]).

We may assume that $\Omega_{2}$ is defined in a neighborhood of 0 by:

$$
\Omega_{2} \cap U_{2}=\left\{z \in U_{2}: r_{2}(z)=\operatorname{Im} z_{0}+\varphi\left(\operatorname{Re} z_{0}, z^{\prime}\right)<0\right\}
$$

where $\varphi$ is a smooth convex function.

Following [11] we consider the point $q_{\nu}^{0}$ in $\partial \Omega_{2}$ closest to $q^{\nu}$. The complex line passing through $q^{\nu}$ and $q_{\nu}^{0}$ is denoted by $l_{0}^{\nu}$, and we set $\tau_{0}^{\nu}=d\left(q^{\nu}, q_{\nu}^{0}\right)$. Then we consider the orthogonal complement of $l_{0}$ through $q_{\nu}$ in $\mathbb{C}^{n+1}$. Since $\Omega_{2}$ is convex of finite type, the distances from $q^{\nu}$ to $\partial \Omega_{2}$ along every complex line in this complement are uniformly bounded and there exists a point $q_{\nu}^{1} \in \partial \Omega_{2}$ where the largest distance is reached; denote by $l_{1}^{\nu}$ the complex line through $q_{\nu}$ and $q_{\nu}^{1}$ and set $\tau_{1}^{\nu}=d\left(q^{\nu}, q_{\nu}^{1}\right)$. Next we consider the orthogonal complement of the complex subspace generated by $l_{1}^{\nu}$ and $l_{2}^{\nu}$, etc. Repeating this process, we obtain orthogonal complex lines $l_{j}^{\nu}, j=0, \ldots, n+1$. Let us consider a unitary transform $U_{\nu}$ of $\mathbb{C}^{n+1}$ such that for any $j$ the image $U_{\nu}\left(l_{j}^{\nu}\right)$ is the coordinate line $z_{j}$ and $U_{\nu}\left(q_{\nu}^{j}\right)$ lies on the imaginary positive axis $I m z_{j}$. Consider also the translation $T_{\nu}$ sending $q^{\nu}$ to the origin and the translation $T_{\nu}^{\prime}$ such that $T_{\nu}^{\prime}(0)=\left(-\tau_{0}^{\nu} i, 0^{\prime}\right)$. The composition $T_{\nu}^{\prime} \circ U_{\nu} \circ T_{\nu}$ gives a new coordinate system centered at $q_{\nu}^{0}$. Note that $d\left(q_{\nu}, \partial \Omega_{2}\right)=\tau_{0}^{\nu} \leq \tau_{j}^{\nu}$ for $j=1, \ldots, n+1$.

We define the dilations

$$
\Lambda_{2, \nu}(z)=\left(\tau_{0}^{\nu} z_{0}, \ldots, \tau_{n}^{\nu} z_{n}\right),
$$

the dilated domains

$$
\Omega_{2}^{\nu}=\left\{z \in\left(\left(\Lambda_{2, \nu}\right)^{-1} \circ T_{\nu}^{\prime} \circ U_{\nu} \circ T_{\nu}\right)\left(U_{2}\right): r_{2} \circ\left(T_{\nu}^{\prime} \circ U_{\nu} \circ T_{\nu}\right)^{-1} \circ \Lambda_{2, \nu}(z)<0\right\}
$$

and the dilated maps

$$
F^{\nu}=\left(\Lambda_{2, \nu}\right)^{-1} \circ T_{\nu}^{\prime} \circ U_{\nu} \circ T_{\nu} \circ f \circ \Lambda_{1, \nu} .
$$

The main point is the normality of the family $\left(F^{\nu}\right)$ which follows by [10]. The next proposition sums up the convergence properties of dilated families. 
Proposition 1.2. (i) The sequence $\left(\Omega_{1}^{\nu}\right)_{\nu}$ converges in the local Hausdorff convergence to the domain $D_{1}=\left\{z \in \mathbb{C}^{n+1}: \operatorname{Im} z_{0}+H\left(z^{\prime}\right)<0\right\}$.

(ii) The sequence $\left(\Omega_{2}^{\nu}\right)_{\nu}$ converges in the local Hausdorff convergence to the domain $D_{2}=\left\{\left(z_{0}, z^{\prime}\right) \in \mathbb{C}^{n+1}:\right.$ Im $\left.z_{0}+P\left(z^{\prime}\right)<0\right\}$ where $D_{2}$ is a convex domain of finite type $\leq 2 \mathrm{~m}$ and $P$ is a convex polynomial of degree $\leq 2 \mathrm{~m}$.

(iii) There exists a rational biholomorphism $\Psi$ from $D_{2}$ to a bounded domain contained in the polydisc (the Cayley transformation in each variable). The image of infinity under this transformation is contained in $\bigcup_{j=0}^{n}\left\{z_{j}=1\right\}$.

(iv) The sequence $\left(F^{\nu}\right)_{\nu}$ converges uniformly on compact subsets of $D_{1}$ to a holomorphic map $F$ from $D_{1}$ to $D_{2}$.

Proof. Part (i) is immediate; (ii) is proved in [10]. Parts (iii) and (iv) follow from the fact that $D_{2}$ is contained in the intersection of $(n+1)$ half-spaces in generic position ([10]).

In order to show the equicontinuity of the family $F^{\nu}$ up to the boundary we need a uniform lower estimate on the Kobayashi infinitesimal metric on the domains $\Omega_{2}^{\nu}$ :

Proposition 1.3. Let $p$ be a point of $\partial D_{2}$. There exists a neighborhood $U$ of $p$ such that:

$$
K_{\Omega_{2}^{\nu}}(z, X) \gtrsim \frac{|X|}{d\left(z, \partial \Omega_{2}^{\nu}\right)^{1 / 2 m}}
$$

on $\left(\Omega_{2}^{\nu} \cap U\right) \times \mathbb{C}^{n+1}$.

Since the sequence of defining functions of $\Omega_{j}^{\nu}$ converges uniformly together with all its derivatives to the defining function of $D_{j}$ and every $\Omega_{2}^{\nu}$ is convex of finite type, a direct examination of the construction of [9] implies that for every real $0<\lambda<1$ there exists a neighborhood $U$ of $p$ such that for any $\nu \geq 1$ and any $\zeta \in \partial \Omega_{2}^{\nu} \cap U$ there exists a function $\varphi_{\nu, \zeta}$ plurisubharmonic on $\Omega_{2}^{\nu} \cap U$, continuous on $\overline{\Omega_{2}^{\nu}} \cap U$ and satisfying:

$$
\begin{gathered}
\left|\varphi_{\nu, \zeta}(z)-\varphi_{\nu, \zeta}(w)\right| \lesssim|z-w|^{\lambda} \\
\varphi_{\nu, \zeta}(z) \lesssim-|z-\zeta|^{2 \lambda m}, \varphi_{\nu, \zeta}(\zeta)=0
\end{gathered}
$$

for $z, w \in \overline{\Omega_{\nu}} \cap U$ (the point is that the constants are independent of $\nu$ ). Then the desired estimate follows from the results of [7].

Proposition 1.4. Suppose that there exists a sequence $\left(a^{\nu}\right)_{\nu}$ in $D_{1}$ converging to $a \in \partial D_{1}$ such that for any $\nu$ one has $a^{\nu} \in \Omega_{1}^{\nu}$ and $\left(F^{\nu}\left(a^{\nu}\right)\right)_{\nu}$ converges to $p \in \partial D_{2}$. Then there exists a neighborhood $V_{0}$ of a such that:

$$
\left|F^{\nu}(z)-F^{\nu}\left(z^{\prime}\right)\right| \lesssim\left|z-z^{\prime}\right|^{1 / 2 m}
$$

on $\overline{\Omega_{1}^{\nu}} \cap V_{0}$

Proof. Propositions 1.1, 3.1 and the well known argument based on the decreasing property of the Kobayashi metric imply the uniform upper estimate on the norm of the differential $d F^{\nu}$. The desired assertion then follows by standard integration (see details in $[6]$ ).

For $z^{0}$ in $\partial D_{1}$, we denote by $C_{D_{1}}\left(F, z^{0}\right)$ the cluster set of the map $F$ at the point $z^{0}$. Proposition 1.1 implies that for sufficiently large $\nu$ we have $\varepsilon_{\nu} \simeq\left(\delta_{\nu}\right)$ and so by the form of the dilations $\Lambda_{1, \nu}$ and $\Lambda_{2, \nu}$ we obtain that $C_{D_{1}}\left(F, z^{0}\right)$ does not intersect 
the domain $D_{2}$ for every $z^{0} \in D_{1}$. Let $S^{c}$ be the set of points $p$ in $\partial D_{1}$ such that $F$ extends continuously to $\partial D_{1}$ in a neighborhood of $p$ and let $S^{\infty}$ denote the set of points $p$ such that $\lim _{z \rightarrow p}|F(z)|=+\infty$. We have the following stratification of $\partial D_{1}$ :

Lemma 1.1. One has $\partial D_{1}=S^{c} \cup S^{\infty}$ and $S^{c}$ is open dense in $\partial D_{1}$.

Proof. Let $p$ be a point in $\partial D_{1}$. Assume that there exists a sequence $\left(p_{\nu}\right)$ in $D_{1}$ such that $\lim _{\nu \rightarrow \infty} f\left(p_{\nu}\right)=q \in \partial D_{2}$. Then it follows by [17] that $p \in S^{c}$. Thus we get the desired stratification. To show the density of $S^{c}$, assume that there exist a point $p$ in $\partial D_{1}$ and a neighborhood $U$ of $p$ such that $U \cap \partial D_{1}$ is contained in $S^{\infty}$. For the map $\Psi$ given by part (iii) of Proposition 1.2 let us consider the function $u(z)=\sum_{j=0}^{n} \log \left|\left(\Psi_{j} \circ F\right)(z)-1\right|$ which is plurisubharmonic on $D_{1}$ and tends to $-\infty$ as $z$ tends to $U \cap \partial D_{1}$. Then $u(z)$ is identically $-\infty$ in $D_{1}$, that is $F$ is $\infty$ in $D_{1}$ : a contradiction.

As usual we say that $F$ extends to an algebraic map in $\mathbb{C}^{n+1}$ if its graph is contained in a complex algebraic variety of dimension $(n+1)$ in $\mathbb{C}^{n+1} \times \mathbb{C}^{n+1}$.

Proposition 1.5. The map $F$ extends to an algebraic map in $\mathbb{C}^{n+1}$.

Proof. Let $p \in \partial D_{1}$ be a strictly pseudoconvex point in $S^{c}$. If $F(p)$ also is a point of strict pseudoconvexity in $\partial D_{2}$, then it follows by [15] that $F$ extends as a local CR diffeomorphism of class $\mathcal{C}^{\infty}$ near $p$. The reflection principle [14] implies that $F$ extends to a local biholomorphism in a neighborhood of $p$ and therefore $F$ extends to an algebraic map by [18].

Thus it suffices to prove that the image of $U \cap \partial D_{1}$ (where $U$ is a neighborhood of $p$ in $\left.\mathbb{C}^{n+1}\right)$ is not contained in the set of weakly pseudoconvex points $\omega\left(\partial D_{2}\right)$ of $\partial D_{2}$. Indeed, assume by contradiction that $F\left(U \cap \partial D_{1}\right)$ is contained in $\omega\left(\partial D_{2}\right)$. According to [5] we get the following local stratification of $\omega\left(\partial D_{2}\right): \omega\left(\partial D_{2}\right)=M_{1} \cup \ldots \cup M_{s}$ where every $M_{j}$ is a smooth variety of holomorphic dimension zero. It follows by [16] that every $M_{j}$ is contained in a smooth strictly pseudoconvex hypersurface $S_{j}$. Hence, in view of the continuity of $F$, there exist a point $a \in \partial D_{1}$ and a neighborhood $V$ of $a$ in $\mathbb{C}^{n+1}$ such that $F\left(V \cap \partial D_{1}\right)$ is contained in $S_{j_{0}}$ for certain $j_{0}$. Therefore, $F$ extends to a local diffeomorphism between $\partial D_{1}$ and $S_{j_{0}}$ near $a$ by [15]. This is a contradiction.

\section{Proof of Theorem A (part (i))}

We assume now that $\Gamma_{1}$ and $\Gamma_{2}$ are smooth convex hypersurfaces of finite type in $\mathbb{C}^{n+1}$ and $f$ is a Lipschitz map.

Lemma 2.1. The sequence $\left(F^{\nu}(0)\right)_{\nu}$ is bounded.

Proof. Since $f$ is Lipschitz we have $\left|q_{\nu}\right| \lesssim \delta_{\nu}$; it follows by Proposition 1.1 that $\tau_{0}^{\nu} \simeq \delta_{\nu}$. Hence $\left|T_{\nu}^{\prime} \circ U_{\nu} \circ T_{\nu}(0)\right| \lesssim \delta_{\nu}$ and we get the assertion by definition of $F^{\nu}$.

Without loss of generality we may assume now that $\left(F^{\nu}(0)\right)_{\nu}$ converges to $p \in$ $\partial D_{2}$. It follows by the continuity of $F^{\nu}$ up to the boundary that there exists a sequence $\left(a^{\nu}\right)_{\nu}$ in $D_{1}$ such that $a^{\nu} \in \Omega_{1}^{\nu}$ for any $\nu$ and $\lim _{\nu \rightarrow \infty} F^{\nu}\left(a^{\nu}\right)=p$. Hence, one can apply Proposition 1.4 and pass to the limit as $\nu \longrightarrow \infty$ in the Hölder estimate. We obtain that there exists a neighborhood $U$ of 0 such that $F$ extends to a continuous map up to $\overline{D_{1}} \cap U$ with $F\left(\partial D_{1} \cap U\right) \subset \partial D_{2}$ and $F(0)=p$. Moreover, 
$F$ is locally finite-to-one near the boundary in view of $[17]$ (see also $[6,7]$ ). In particular, $F$ is nondegenerate i.e. the jacobian of $F$ does not vanish identically.

Lemma 2.2. The pull-back $F^{-1}(p)$ is finite near 0 .

We recall that if $M$ is a real analytic hypersurface in $\mathbb{C}^{n+1}$ of the form $M=$ $\left\{z \in \mathbb{C}^{n+1}: r(z, \bar{z})=0, r=\bar{r}, d r(z, \bar{z}) \neq 0\right\}$, the Segre surface associated with $M$ and a point $\zeta$ in $\mathbb{C}^{n+1}$ is defined by $S_{M}(\zeta)=\left\{z \in \mathbb{C}^{n+1}: r(z, \bar{\zeta})=0\right\}$. We denote by $S_{j}(\zeta)$ the Segre surface associated with $\partial D_{j}$ and by $\hat{S}_{j}(\zeta)$ its projective closure in the complex projective space $\mathbb{P}^{n+1}$. Note that in our case every $S_{j}(\zeta)$ (resp. $\hat{S}_{j}(\zeta)$ ) is an (irreducible) complex algebraic (resp. projective) variety. We will denote by $\mathbb{P}_{\infty}^{n}$ the difference $\mathbb{P}^{n+1} \backslash i\left(\mathbb{C}^{n+1}\right)$, where $i: \mathbb{C}^{n+1} \longrightarrow \mathbb{P}^{n+1}$ is the canonical embedding.

Proof. Let $A$ be a (irreducible) complex algebraic variety containing the graph of $F$ and $\hat{A}$ be its projective closure in $\mathbb{P}^{n+1} \times \mathbb{P}^{n+1}$. Then $\hat{A}$ is a projective (irreducible) variety of dimension $(n+1)$ in $\mathbb{P}^{n+1} \times \mathbb{P}^{n+1}$ and we may consider the natural projections $\pi_{1}: \hat{A} \rightarrow \mathbb{P}^{n+1} \supset i\left(D_{1}\right)$ and $\pi_{2}: \hat{A} \rightarrow \mathbb{P}^{n+1} \supset i\left(D_{2}\right)$. Since $F$ is a nondegenerate map, $\pi_{2} \circ \pi_{1}^{-1}: \mathbb{P}^{n+1} \rightarrow \mathbb{P}^{n+1}$ is a proper algebraic correspondence ([12]). Then there exists an algebraic variety $\Sigma$ of dimension $<(n+1)$ in $\hat{A}$ such that the restrictions of $\pi_{1}$ and $\pi_{2}$ to $\hat{A} \backslash \Sigma$ are locally biholomorphic. For $j=1,2$, the sets $\Sigma_{j}=\pi_{j}(\Sigma)$ are complex projective varieties of dimensions $<(n+1)$ in $\mathbb{P}^{n+1}$. Assume that the set $F^{-1}(p)$ is not compact. Therefore there exists a real algebraic curve $\gamma$ contained in $\pi_{1} \circ \pi_{2}^{-1}(p) \cap \partial D_{1}$. Let $q$ be a regular point of $\gamma$, and $\left(\zeta_{0}(t), \ldots, \zeta_{n}(t)\right)$ be a local parametrization of $\gamma$ near $q$. Consider a real analytic function $h$ defined on $\mathbb{C}^{n} \times[0,1]$ by:

$$
h\left(z^{\prime}, t\right)=-\frac{\overline{\zeta_{0}(t)}}{2 i}+H\left(z^{\prime}, \overline{\zeta^{\prime}(t)}\right)
$$

where $H$ is given by Proposition 1.2 (i).

If $\frac{\partial h}{\partial t}$ is identically zero on $\mathbb{C}^{n} \times[0,1]$, then for every $t$ in $[0,1]$ we get $S_{1}(\zeta(t))=$ $S_{1}(q)$. Since the polynomial $H$ contains no pluriharmonic terms, $H\left(0, \overline{\zeta^{\prime}(t)}\right)$ vanishes identically and $\zeta_{0}(t)=q_{0}$ for every $t \in[0,1]$. For $j \leq n$ let $H_{j}$ denote the restriction of $H\left(z^{\prime}, \overline{\zeta^{\prime}(t)}\right)$ on the complex line $z_{j}$. The polynomial $H_{j}$ contains a term of the form $a z_{j}^{s}{\overline{\zeta_{j}(t)}}^{m_{j}-s}$ with $a \neq 0$ and $s>0$. By assumption this term is identically equal to $a z_{j}^{s} \overline{q_{j}} m_{j}-s$ when $t$ runs over $[0,1]$. Then $\zeta_{j}(t)$ is identically $q_{j}$, which contradicts the regularity of the point $q \in \gamma$. Therefore the family of Segre surfaces $\left(S_{1}(\gamma(t))\right)_{t}$ fills a real hypersurface $M$ in $\mathbb{C}^{n+1}$. Let $\tilde{D}$ be a (simply connected) domain in $\mathbb{C}^{n+1}$ which does not contain the points of $\Sigma_{1} \cup \pi_{1} \circ \pi_{2}^{-1}\left(\mathbb{P}_{\infty}^{n}\right)$ and the intersection $\tilde{D} \cap M$ is not empty. Then the graph of the correspondence $\pi_{2} \circ \pi_{1}^{-1}$ is represented over $\tilde{D}$ as the union of the graphs of holomorphic maps $G_{0}, \ldots, G_{n}$ defined on $\tilde{D}$. We claim that there exists an integer $j$ such that for every $\zeta$ in $\gamma$ near $q$ the set $G_{j}\left(S_{1}(\zeta) \cap \tilde{D}\right)$ is contained in $S_{2}(p)$. It suffices to show that for every $\zeta$ in $\gamma$ near $q$ and $z$ in $S_{1}(\zeta)$ the intersection $\pi_{2} \circ \pi_{1}^{-1}(z) \cap S_{\partial D_{2}}(p)$ is not empty. Let $\zeta$ be a point in $\gamma$ and $\left(a^{\nu}\right)_{\nu}$ a sequence of strictly pseudoconvex points in $\partial D_{1} \backslash \Sigma_{1}$, converging to $\zeta$, such that $F\left(a^{\nu}\right)$ is strictly pseudoconvex in $\partial D_{2}$. Then, according to [15] and the reflection principle [14], $F$ extends locally as a biholomorphism near $a^{\nu}$. Hence the varieties $F\left(S_{1}\left(a^{\nu}\right)\right)$ and $S_{2}\left(F\left(a^{\nu}\right)\right)$ coincide locally in view of the biholomorphic 
invariance of Segre surfaces (see [18]). Since $\hat{S}_{1}\left(F\left(a^{\nu}\right)\right)$ is irreducible, it is contained in $\pi_{1} \circ \pi_{2}^{-1}\left(\hat{S}_{2}\left(F\left(a^{\nu}\right)\right)\right)$. Therefore, for every $z$ in $S_{1}\left(a^{\nu}\right)$ the intersection $\pi_{2} \circ \pi_{1}^{-1}(z) \cap$ $\hat{S}_{2}\left(F\left(a^{\nu}\right)\right)$ is not empty. Since each point $z$ in $S_{1}(\zeta)$ is a cluster point for a sequence $z^{\nu}$ in $S_{1}\left(a^{\nu}\right)$, the intersection $\pi_{2} \circ \pi_{1}^{-1}(z) \cap \hat{S}_{2}(F(p))$ is not empty. Hence for every $\zeta$ there exists an integer $j_{\zeta}$ such that $G_{j_{\zeta}}\left(S_{1}(\zeta) \cap \tilde{D}\right)$ is contained in $S_{2}(p)$. By continuity there exists $j$ such that $G_{j}(M \cap \tilde{D})$ is contained in $S_{2}(p)$. Since $M$ is a uniqueness set, $G_{j}(\tilde{D})$ is contained in $S_{2}(p)$. Then $S_{2}(p)$ contains a component of $\pi_{2} \circ \pi_{1}^{-1}(\tilde{D})$ : a contradiction since $\hat{A}$ is irreducible.

We can prove now Theorem A part (i):

Proof of Theorem A (part (i)). We denote by $\mathbb{B}$ the unit ball. Suppose by contradiction that for every $\varepsilon>0$ the intersection $f^{-1}(0) \cap \varepsilon \partial \mathbb{B} \cap \Gamma_{1}$ is not empty. Applying our scaling construction, we get that for every $\varepsilon>0$ and every $\nu$ the intersection $\left(F^{\nu}\right)^{-1}\left(F^{\nu}(0)\right)$ is not empty. One can assume by Lemma 2.1 that the sequence $\left(F^{\nu}(0)\right)$ converges to a (finite) point $p \in \partial D_{2}$. It follows from Proposition 1.4 that $F^{-1}(p)$ intersects $\varepsilon \partial \mathbb{B} \cap \partial D_{1}$ for every $\varepsilon>0$. This contradicts Lemma 2.2 .

\section{Proof of Theorem A (Part (ii))}

We suppose now that $f$ is a holomorphic map from a strictly pseudoconvex domain $\Omega_{1}$ to a convex domain of finite type $\Omega_{2}$, defined near 0 , continuous up to $\partial \Omega_{1}$ with $f(0)=0$. We keep the same notations as in the previous sections and we apply the scaling method. The limit domain $D_{1}$ is now the unbounded representation of the unit ball in $\mathbb{C}^{n+1}$ :

$$
\mathbb{H}=\left\{z \in \mathbb{C}^{n+1}: I m z_{0}+\left|z^{\prime}\right|^{2}<0\right\} .
$$

Proposition 3.1. (i) The hypersurface $\partial D_{2}$ is strictly pseudoconvex near every finite point.

(ii) The map $F$ is a biholomorphism from $\mathbb{H}$ to $D_{2}$.

Proof. The proof is similar to [6] since we already proved in Proposition 1.5 that $F$ extends to an algebraic map.

The boundary behavior of $F$ is given by the following proposition:

Proposition 3.2. The map $F$ extends to a homeomorphism from $\overline{\mathbb{H}} \cup\{\infty\}$ to $\overline{D_{2}} \cup$ $\{\infty\}$.

After a global biholomorphic polynomial change of coordinates one can assume that the polynomial $P$ does not contain purely harmonic terms.

Consider the translations $L_{t}\left(z_{0}, z^{\prime}\right)=\left(z_{0}+t, z^{\prime}\right), t \in \mathbb{R}$. Then the transformations $h_{t}=F^{-1} \circ L_{t} \circ F$ form a one-parameter subgroup of Aut(HI). Fix a Cayley biholomorphism $(\mathrm{Ca})$ from $\mathbb{H}$ to $\mathbb{B}$ and consider the one-parameter group $\left(g_{t}\right)$ of transformations of the unit ball $\mathbb{B}$ defined by $g_{t}=(C a) \circ h_{t} \circ(C a)^{-1}$. Applying to $G=(C a) \circ F^{-1}$ the argument similar to [1] we obtain that $\left(g_{t}\right)_{t}$ (and hence $\left.\left(h_{t}\right)_{t}\right)$ is a parabolic group, i.e. that there exists a point $p$ in $\partial \mathbb{H} \cup\{\infty\}$ such that for every $z$ in $\mathbb{H}$ one has $\lim _{t \rightarrow \pm \infty} h_{t}(z)=p$.

Let us consider now the Cayley transform $(\tilde{C a})$ such that $(\tilde{C a})^{-1} \circ(C a)(p)=$ $0 \in \partial \mathbb{H}$. If $\tilde{G}$ is the map $\tilde{G}=(\tilde{C} a)^{-1} \circ G$, the one-parameter group $\tilde{h}_{t}=\tilde{G} \circ L_{t} \circ \tilde{G}^{-1}$ 
is parabolic and for every $z$ in $\mathbb{H}$ we have $\lim _{t \rightarrow \pm \infty} \tilde{h}_{t}(z)=0 \in \partial \mathbb{H}$. Consequently $\tilde{h}_{t}=\left(\tilde{h}_{t}^{0}, \tilde{h}_{t}^{\prime}\right)$ is an automorphism of $\mathbb{H}$ of the form:

$$
\begin{gathered}
\tilde{h}_{t}^{0}(z)=\frac{\rho_{t} z_{0}}{1-\left[2 i\left\langle z^{\prime}, a_{t}\right\rangle+\left(r_{t}+i\left\langle a_{t}, a_{t}\right\rangle\right) z_{0}\right]}, \\
\tilde{h}_{t}^{\prime}(z)=\frac{A_{t}\left(z^{\prime}+z_{0} a_{t}\right)}{1-\left[2 i\left\langle z^{\prime}, a_{t}\right\rangle+\left(r_{t}+i\left\langle a_{t}, a_{t}\right\rangle\right) z_{0}\right]}
\end{gathered}
$$

where $\rho_{t}$ and $r_{t}$ are real numbers, $a_{t}$ is a complex number and $A_{t}$ is an $(n \times n)$ invertible matrix and $\langle.,$.$\rangle denote the scalar product in \mathbb{C}^{n}$.

Lemma 3.1. For every $z$ in $\mathbb{C}^{n+1}$, we have

$$
\tilde{G}(z)=\left(\tilde{G}_{0}, \tilde{G}^{\prime}\right)=\left(-\left(c z_{0}+\gamma\left(z^{\prime}\right)\right)^{-1}, g\left(z^{\prime}\right)\left(c z_{0}+\gamma\left(z^{\prime}\right)\right)^{-1}\right)
$$

where $c$ is a complex constant, $\gamma$ is a polynomial and $g$ is a polynomial automorphism of $\mathbb{C}^{n}$.

Proof. Since $\left(\tilde{h}_{t}\right)_{t}$ is a one-parameter group we have the following equations:

$$
\left\{\begin{aligned}
A_{t} & =e^{t A} \\
\rho_{t} & =e^{\rho t} \\
a_{t+s} & =a_{s}+A_{s}^{*} a_{t}, \\
r_{t+s} & =r_{s}-2 \operatorname{Im}\left\langle A_{s} a_{s}, a_{t}\right\rangle+\rho_{s} r_{t}
\end{aligned}\right.
$$

where $A$ is an $(n \times n)$ matrix and $A^{*}$ is obtained by the transposition and complex conjugation of $A$. Since $A_{t}^{*} A_{t}=\rho_{t} I$ where $I$ is the identity matrix, we obtain the equality $A+A^{*}=\rho I$ and finally $A^{*}=\frac{A^{*}-A}{2}+\frac{\rho}{2} I$. Hence $A^{*}$ is diagonalizable in an orthonormal basis.

If we differentiate the third equation of this system (in $t$ ), we obtain that $a_{s}=$ $s \alpha+\left(e^{s A}-I\right) \beta$ where $\alpha=\left(\alpha_{1}, \ldots, \alpha_{n}\right), \beta=\left(\beta_{1}, \ldots, \beta_{n}\right) \in \mathbb{C}^{n}$. Then if we consider the last equation and if we assume that $\rho$ is not equal to 0 , we get the identity:

$$
1-\left[2 i\left\langle z^{\prime}, t \alpha+\left(e^{t A^{*}}-I\right) \beta\right\rangle+\left(r_{t}+i a_{t}^{2}\right) z_{0}\right]=O\left(e^{\rho t}\right)
$$

which contradicts the condition: $\lim _{t \rightarrow \pm \infty} \tilde{h_{t}^{0}}(z)=0$. Consequently $\rho=0$ and so $\rho_{s}=1$. Then we obtain by differentiating (in $t$ ) the last equation that:

$$
r_{s}=\left\langle 2 \operatorname{Im} \beta, e^{s A^{*}} \beta\right\rangle+[r-2 \operatorname{Im}\langle A \beta, \beta\rangle] s
$$

where $r \in \mathbb{R}$.

Then we have:

$$
\begin{aligned}
\tilde{G}_{0}\left(z_{0}+t, z^{\prime}\right) & =\frac{\tilde{G}_{0}\left(z_{0}, z^{\prime}\right)}{1-\left[2 i\left\langle\tilde{G}^{\prime}(z), a_{t}\right\rangle+\left(r_{t}+i a_{t}^{2}\right) \tilde{G}_{0}(z)\right]}, \\
\tilde{G}^{\prime}\left(z_{0}+t, z^{\prime}\right) & =\frac{e^{t A}\left(\tilde{G}^{\prime}(z)+a_{t} \tilde{G}_{0}(z)\right)}{1-\left[2 i\left\langle\tilde{G}^{\prime}(z), a_{t}\right\rangle+\left(r_{t}+i a_{t}^{2}\right) \tilde{G}_{0}(z)\right]} .
\end{aligned}
$$

After successive differentiation (in $t$ ) and integration (in $z_{0}$ ) of this equalities we obtain:

$$
-\frac{1}{\tilde{G}_{0}\left(z_{0}, z^{\prime}\right)}=i z_{0}^{2} \alpha^{2}+\left[r+2 i\langle A \beta, \beta\rangle+2 i\left\langle h\left(z^{\prime}\right), \alpha\right\rangle\right] z_{0}+2 i\left\langle e^{A z_{0}} h\left(z^{\prime}\right), \beta\right\rangle+\gamma\left(z^{\prime}\right)
$$

where $\gamma$ is a holomorphic function on $\mathbb{C}^{n}$ and $h: \mathbb{C}^{n}: \longrightarrow \mathbb{C}^{n}$ is a holomorphic map. Since $\tilde{G}$ is a biholomorphism from $D_{2}$ to $\mathbb{H}$, the domain $D_{2}$ is contained in the 
domain $\left\{\operatorname{Im}\left(-\frac{1}{\tilde{G}_{0}}\right)<0\right\}$. Hence, considering points $z$ with $\left|R e z_{0}\right|$ large enough in the previous equation we obtain that $\alpha=0$. Since the image $\tilde{G}\left(\partial D_{2}\right)$ is contained in $\partial \mathbb{H} \cup\{\infty\}$, we get by considering a point $\left(t-i P\left(z^{\prime}\right), z^{\prime}\right)$ in $\partial D_{2}$ :

$$
\begin{aligned}
-[r & +2 i\langle A \beta, \beta\rangle] P\left(z^{\prime}\right)+\operatorname{Im} \gamma\left(z^{\prime}\right)+\beta^{2}+\left|e^{-i A P\left(z^{\prime}\right)} h\left(z^{\prime}\right)\right|^{2} \\
& \left.=-4 \operatorname{Re}\left\langle e^{A\left(t-i P\left(z^{\prime}\right)\right.}\right) h\left(z^{\prime}\right), \beta\right\rangle .
\end{aligned}
$$

This equality implies that the function $h_{j}\left(z^{\prime}\right) \bar{\beta}_{j}$ is identically zero on $\mathbb{C}^{n}$ for every $j$. Since $\tilde{G}$ is algebraic, each of its components and the restrictions of these components to complex lines are algebraic. In particular for every $z^{\prime}$ in $\mathbb{C}^{n}$, the map defined on $\mathbb{C}$ by $z_{0} \mapsto-\frac{\beta+e^{A z_{0}} h\left(z^{\prime}\right)}{c z_{0}+\gamma\left(z^{\prime}\right)}$ is algebraic. So it is for the map $z_{0} \mapsto e^{A z_{0}} h\left(z^{\prime}\right)$. Then this map does not depend on $z_{0}$. We denote $g$, the map defined on $\mathbb{C}^{n}$, by $g\left(z^{\prime}\right)=-\left(\beta+e^{A z_{0}} h\left(z^{\prime}\right)\right)$.

We obtain that $\tilde{G}_{0}(z)=-\left(c z_{0}+\gamma\left(z^{\prime}\right)\right)^{-1}$ and $\tilde{G}^{\prime}(z)=g\left(z^{\prime}\right)\left(c z_{0}+\gamma\left(z^{\prime}\right)\right)^{-1}$ where $c$ is a complex constant.

Since the set $\tilde{G}\left(\partial D_{2}\right)$ is contained in $\partial \mathbb{H} \cup\{\infty\}$, we get

$$
-c P\left(z^{\prime}\right)+\operatorname{Im} \gamma\left(z^{\prime}\right)+\left|g\left(z^{\prime}\right)\right|^{2}=0 .
$$

Considering the non pluriharmonic terms we obtain that the polynomial $P$ is nonnegative and:

$$
\left|g\left(z^{\prime}\right)\right| \lesssim\left(P\left(z^{\prime}\right)\right)^{1 / 2},\left|\gamma\left(z^{\prime}\right)\right| \lesssim\left(P\left(z^{\prime}\right)\right)^{1 / 2}
$$

Since $\Phi(z)=\left(-\frac{1}{z_{0}}, \frac{z^{\prime}}{z_{0}}\right) \in \operatorname{Aut}(\mathbb{H})$, the map $\Phi \circ \tilde{G}$ is a biholomorphism from $D_{2}$ to $\mathbb{H}$ of the form $(\Phi \circ \tilde{G}(z))=\left(c z_{0}+\gamma\left(z^{\prime}\right), g\left(z^{\prime}\right)\right)$. This shows that $g$ is an automorphism of $\mathbb{C}^{n}$. Then the above estimates on $g$ and $\gamma$ imply that $\gamma$ is a holomorphic polynomial function and $g$ is a polynomial automorphism on $\mathbb{C}^{n}$, which gives Lemma 3.1.

Proof of Proposition 3.1. It suffices to prove that $(\tilde{C a}) \circ \tilde{G}$ extends to a homeomorphism from $D_{2} \cup\{\infty\}$ to $\overline{\mathbb{B}}$. Obviously $(\tilde{C a}) \circ \tilde{G}$ extends to $\partial \tilde{D}_{2}$ as a local homeomorphism in a neighborhood of every finite point $p$ in $\partial \tilde{D}_{2}$. On the other hand, it follows from obtained estimates on $g$ and $\gamma$ that $\lim _{\substack{|z| \rightarrow \infty \\ z \in D_{2}}}(\tilde{C} a \circ \tilde{G})(z)=(\tilde{C a})(0)$.

Now part (ii) of Theorem A follows from Propositions 1.4 and 3.2 quite similarly to the proof of part (i) (see also [6]).

\section{REFERENCES}

1. E.Bedford, S.Pinchuk, Domains in $\mathbb{C}^{2}$ with noncompact automorphism group, Math.USSR Sbornik 63 (1989), 141-151. MR 92f:32024

2. E.Bedford, S.Pinchuk, Convex domains with noncompact automorphism group, Matem. Sb. 185-5 (1994), 3-26. MR 95e:32037

3. S.Bell, Local regularity of CR homeomorphisms, Duke Math. J. 57 (1988), 295-300. MR 89f: 32026

4. S.Bell, D.Catlin, Regularity of CR mappings, Math. Zeit. 199 (1988), 357-368. MR 89i:32028

5. D.Catlin, Boundary invariants of pseudoconvex domains, Ann. Math. 120 (1984), 529-586. MR 86c:32019

6. B.Coupet, S.Pinchuk, A.Sukhov, On boundary rigidity and regularity of holomorphic mappings, Intern. J. Math. 7-5 (1996), 617-643. MR 97k:32043

7. B.Coupet, A.Sukhov, On CR mappings between pseudoconvex hypersurfaces of finite type in $\mathbb{C}^{2}$, Duke Math. J. 88-2 (1997), 281-304. MR 98d:32031 
8. K.Diederich, G.Herbort, Pseudoconvex domains of semiregular type, Contributions to complex analysis and analytic geometry, Vieweg Braunschweig (1994), 127-161. MR 96b:32019

9. J.E.Fornaess, N.Sibony, Construction of P.S.H. functions on weakly pseudoconvex domains, Duke Math. J. 58 (1989), 633-656. MR 90m:32034

10. H.Gaussier, Characterization of convex domains with noncompact automorphism group, Mich. J. Math. 44-2 (1997), 375-388. CMP 97:15

11. J.D.McNeal, Convex domains of finite type, J. Funct. Anal. 108 (1992), 361-373. MR 93h:32020

12. D.Mumford, Algebraic geometry I. Complex projective varieties, Springer Verlag (1976). MR 56:11992

13. L.Nirenberg, S.Webster, P.Yang, Local boundary regularity of holomorphic mappings, Comm. Pure Appl. Math. 33 (1980), 305-338. MR 81g:32020

14. S.Pinchuk, On the analytic continuation of holomorphic mappings, Math. USSR Sbornik $\mathbf{2 7}$ (1975), 375-392.

15. S.Pinchuk, Sh. Tsyganov, Smoothness of CR mappings between strictly pseudoconvex hypersurfaces, Math. USSR Izvestija 35 (1990), 457-467. MR 90j:32022

16. N.Sibony, Une classe de domaines pseudoconvexes, Duke Math. J. 55-2 (1987), 299-319. MR 88g:32036

17. A.Sukhov, On boundary regularity of holomorphic mappings, Russian Acad. Sci. Sb. Math. 83-2 (1995), 541-551.

18. S.Webster, On the mapping problem for algebraic real hypersurfaces, Invent. math. 43 (1977), 53-68. MR 57:3431

CMi (Center of Mathematics \& Information), University of Provence, 39 rue JoliotCurie, 13453 Marseille Cedex 13, France 